\title{
Kaempferol suppresses lipid accumulation in macrophages through the downregulation of cluster of differentiation 36 and the upregulation of scavenger receptor class $B$ type I and ATP-binding cassette transporters A1 and G1
}

\author{
XIU-YING LI, LING-XI KONG, JUAN LI, HAI-XIA HE and YUAN-DA ZHOU
}

Department of Pharmacy, The First Affiliated Hospital of Chongqing Medical University, Chongqing 400010, P.R. China

Received August 26, 2012; Accepted October 22, 2012

DOI: $10.3892 / \mathrm{ijmm} .2012 .1204$

\begin{abstract}
The accumulation of foam cells in atherosclerotic lesions is a hallmark of early-stage atherosclerosis. Kaempferol has been shown to inhibit oxidized low-density lipoprotein (oxLDL) uptake by macrophages; however, the underlying molecular mechanisms are not yet fully investigated. In this study, we shown that treatment with kaempferol markedly suppresses oxLDL-induced macrophage foam cell formation, which occurs due to a decrease in lipid accumulation and an increase in cholesterol efflux from THP-1-derived macrophages. Additionally, the kaempferol treatment of macrophages led to the downregulation of cluster of differentiation 36 (CD36) protein levels, the upregulation of ATP-binding cassette (ABC) transporter A1 (ABCA1), scavenger receptor class B type I (SR-BI) and ABCG1 protein levels, while no effects on scavenger receptor A (SR-A) expression were observed. Kaempferol had similar effects on the mRNA and protein expression of ABCA1, SR-BI, SR-A, CD36 and ABCG1. The reduced CD36 expression following kaempferol treatment involved the inhibition of c-Jun-activator protein-1 (AP-1) nuclear translocation. The inhibition of AP-1 using the inhibitor, SP600125, confirmed this involvement, as the AP-1 inhibition significantly augmented the kaempferol-induced reduction in CD36 expression. Accordingly, the kaempferol-mediated suppression of lipid accumulation in macrophages was also augmented by SP600125. The increased expression of ABCA1, SR-BI and ABCG1 following kaempferol treatment was accompanied by the enhanced protein expression of heme oxygenase-1 (HO-1).
\end{abstract}

Correspondence to: Professor Yuan-da Zhou, Department of Pharmacy, The First Affiliated Hospital of Chongqing Medical University, 1 You Yi Road, Yuan Jia Gang, Chongqing 400016, P.R. China

E-mail: zhouyuanda001@163.com

Key words: kaempferol, cluster of differentiation 36, ATP-binding cassette transporter A1, ATP-binding cassette transporter G1, scavenger receptor class B type I, heme oxygenase-1, activator protein-1
This increase was reversed following the knockdown of the HO-1 gene using small hairpin RNA (shRNA). Moreover, the kaempferol-mediated attenuation of lipid accumulation and the promotion of cholesterol efflux was also inhibited by HO-1 shRNA. In conclusion, the c-Jun-AP-1-dependent downregulation of CD36 and the HO-1-dependent upregulation of ABCG1, SR-BI and ABCA1 may mediate the beneficial effects of kaempferol on foam cell formation.

\section{Introduction}

The pathogenesis of atherosclerosis is complex and involves the impact of many well-documented traditional risk factors (1). Among these, the accumulation of macrophage foam cells in the intima is a hallmark of early-stage atherosclerosis (2). It is well established that foam cells are mainly derived from macrophages due to the continuous uptake of modified low-density lipoprotein (LDL), leading to excessive lipoprotein-derived cholesterol accumulation inside the cells $(3,4)$. Once foam cells are formed, the development of atherosclerosis can be accelerated through plaque disruption (2). Hence, reducing the formation of foam cells may be an efficient strategy for the treatment and prevention of atherosclerosis. The cholesterol homeostasis in macrophages has been reported to be mainly regulated by cholesterol internalization and cholesterol efflux through the so-called scavenger receptors and reverse cholesterol transporters. Of the many cell surface proteins, scavenger receptor A (SR-A) and cluster of differentiation 36 (CD36) have been shown to be responsible for the uptake of modified lipoproteins by human blood monocyte-derived macrophages (HMDMs) (5). By contrast, the efflux of cholesterol is regulated by reverse cholesterol transporters (RCTs) including scavenger receptor class B type I (SR-BI), ATP-binding cassette (ABC) transporter A1 and ABCG1 (6,7). Therefore, a therapeutic strategy involving the upregulation of the expression of RCTs or the downregulation SR expression, resulting in the reduction of cholesterol accumulation in macrophages would be highly desirable for the treatment of atherosclerosis.

Flavonoids are normal constituents of the human diet and are known to have anti-inflammatory potential, as well as antioxidant properties both in animal and human models $(8,9)$.Kaempferol, a flavonoid present in various natural sources including tea, 
cabbage, beans, tomatoes, onions, leeks and apples, has been shown to exert several beneficial effects in cardiovascular and nerve systems, such as the reduction of hydroxyl radicals (10), and inflammatory response in macrophage cells (11). Current research indicates that kaempferol ameliorates the development of atherosclerosis in apolipoprotein E-deficient mice (12); however, the mechanisms responsible for the anti-atherogenic effects of kaempferol are not yet fully defined. As mentioned above, SRs and RCTs are potential molecular targets for the selective delivery of anti-atherosclerosis agents to foam cells. Therefore, we hypothesized that the anti-atherogenic effects of kaempferol are related to the suppression of foam cell formation by regulating the expression of SRs and RCTs. Furthermore, a number of studies have reported that heme oxygenase-1 (HO-1), the critical enzyme in heme catabolism, or activator protein-1 (AP-1) mediates the antioxidative or anti-inflammatory properties of kaempferol, respectively $(13,14)$. However, whether HO-1 or AP-1 contributes to the suppression of the formation of foam cells by kaempferol warrants further investigation.

The present study was designed to investigate whether kaempferol inhibits the formation of foam cells and explore the molecular mechanisms involved. Our results suggest that kaempferol suppresses macrophage-derived foam cell formation via the HO-1-dependent upregulation of RCT expression and the c-Jun (a subunit of AP-1)-dependent downregulation of CD36 expression.

\section{Materials and methods}

Cell culture and transfection. The THP-1 human monocytederived cell line was purchased from the American Type Culture Collection (ATCC, Manassas, VA, USA), and cultured in RPMI-1640 medium containing $10 \%$ fetal bovine serum (FBS), $20 \mathrm{~g} / \mathrm{ml}$ streptomycin and $20 \mathrm{IU} / \mathrm{ml}$ penicillin at $37^{\circ} \mathrm{C}$ in $5 \% \mathrm{CO}_{2}$. Differentiation into macrophages was achieved by treating the cells with phorbol 12-myristate 13-acetate (PMA, $200 \mathrm{ng} / \mathrm{ml}$ ) for $48 \mathrm{~h}$. Reagents for cell culture were purchased from Invitrogen (Carlsbad, CA, USA). Cell transfections were performed with the SuperFect fragment (Qiagen, Valencia, CA, USA) according to the manufacturer's instructions using scrambled or HO-1 small hairpin RNA (shRNA) in a 6-well plate or 50-ml flask. Cells were incubated for $24 \mathrm{~h}$ after transfection and used for the indicated experiments.

Reagents. PMA and kaempferol (purity, 98.0\%) (Fig. 1) were obtained from Sigma (St. Louis, MO, USA). Mouse antiABCA1, rabbit anti-ABCG1, rabbit anti-CD36 and rabbit anti-SR-BI antibodies were from Abcam (Cambridge, MA, USA), and goat anti-SR-A antibody was from Santa Cruz Biotechnology, Inc. (Santa Cruz, CA, USA). Rabbit anti-HO-1, rabbit anti-c-Fos, rabbit anti-c-Jun antibodies were purchased from Cell Signaling Technology (Beverly, MA, USA). Scrambled and HO-1 shRNAs were purchased from Shanghai GenePharma Co., Ltd. (Shanghai, China). 3-DodecanoylNBD-cholesterol was obtained from Cayman Chemical Co. (Ann Arbor, MI, USA).

Cell viability assay with methyl thiazoyltetrazolium (MTT). Macrophages were seeded at density of $7.5 \times 10^{4}$ cells/well in 96-well plates and cell viability was determined by MTT

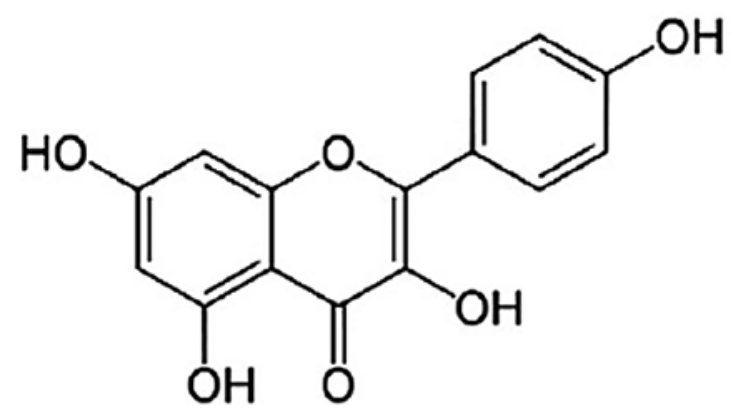

Figure 1. Chemical structure of kaempferol.

assay. MTT assay was performed as described in our previous study (15). Briefly, following treatment with or without kaempferol for $24 \mathrm{~h}$, the culture supernatant was removed. The cells were washed with PBS and incubated with $100 \mu \mathrm{l}$ of MTT $(1 \mathrm{mg} / \mathrm{ml})$ in culture medium at $37^{\circ} \mathrm{C}$ for $4 \mathrm{~h}$. The culture medium with the dye was then removed and $150 \mu \mathrm{l}$ of DMSO/ well were added for formazan solubilization. The absorbance of the converted dye was measured at a wavelength of $490 \mathrm{~nm}$ using a Sunrise Remote Microplate Reader (Tecan Austria $\mathrm{GmbH}$, Grödig, Austria). The viability of the macrophages in each well was presented as a percentage of the control cells.

shRNA. Mouse HO-1 (NM_010442.2)-specific oligonucleotides, including 2 complementary 21-nucleotide sequences corresponding to positions 792-812 downstream of the transcription start site (GCTGACAGAGGAACACAAAGA) and separated by a 9-nucleotide non-complementary spacer (TTCAAGAGA), were selected for the targeted suppression of HO-1. A negative control shRNA vector expressing an oligonucleotide containing a 20-nucleotide sequence not targeting HO-1 and separated by a 9-nucleotide non-complementary spacer (CAAGAGATT) from the reverse complement of the same 20-nucleotide sequence was used.

Oil red $O$ staining. Foam cells derived from macrophages were identified by $\mathrm{Oil}$ red $\mathrm{O}$ staining. Oil red $\mathrm{O}$ is a fat-soluble diazo dye used for staining neutral triglycerides and lipids and some lipoproteins (16). After the culture of THP-1-derived macrophages with $100 \mu \mathrm{g} / \mathrm{ml}$ oxidized LDL (oxLDL) in the presence or absence of kaempferol for $24 \mathrm{~h}$, cells were washed once with PBS, fixed in $10 \%$ paraformaldehyde-PBS for $30 \mathrm{~min}$, and stained for $20 \mathrm{~min}$ in $1 \%$ Oil red $\mathrm{O}$ (in $60 \%$ isopropanol). Hematoxylin was used as the counterstain. After washing with PBS for 3 times, macrophages were photographed under a microscope at x400 magnification.

Cholesterol efflux assay. Cholesterol efflux experiments were performed as previously described (17). After $24 \mathrm{~h}$ of treatment with various concentrations of kaempferol, the THP-1-derived macrophages were incubated with the equilibration of NBD-cholesterol $(1 \mu \mathrm{g} / \mathrm{ml})$ for an additional $6 \mathrm{~h}$ in the presence of kaempferol. The NBD-cholesterollabeled cells were incubated in RPMI-1640 medium for $6 \mathrm{~h}$ after washing with PBS. A multilabel counter (Perkin-Elmer Life Sciences, Waltham, MA, USA) was used to detect the fluorescence-labeled cholesterol released from the cells into 


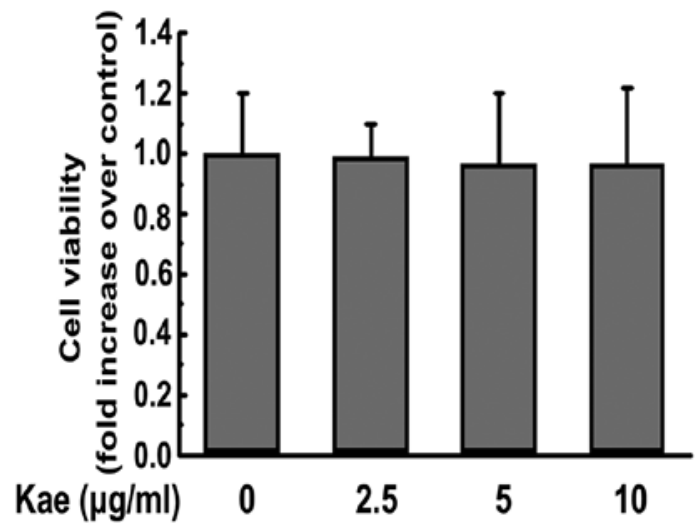

Figure 2. Effect of kaempferol (Kae) on the viability of macrophages. Cells were treated with kaempferol $(2.5,5$ and $10 \mu \mathrm{g} / \mathrm{ml})$ for $24 \mathrm{~h}$ and cell viability was measured by MTT assay. Values are expressed as the means \pm SEM of 3 independent experiments.

the medium. Cholesterol efflux was calculated as a percentage of fluorescence in the medium relative to the total amount of fluorescence.

Quantitative real-time polymerase chain reaction ( $q R T-P C R$ ). Tatol RNA was isolated using RNAiso Plus and was converted into complementary DNA by the PrimeScript RT reagent kit (Perfect Real Time; Takara). Primers used in the qRT-PCR analysis were as follows: mouse SR-BI forward, 5'-ACCCT AACCCAAAGGAGCAT-3' and reverse, 5'-CACAGCAA CGGCAGAACTAC-3'; mouse ABCA1 forward, 5'-CAA TGTCAAGGTGTGGTTCAAT-3' and reverse, 5'-GCTGCTG TTTAGTGAGGTTCAA-3'; mouse CD36 forward, 5'-TCGCT TCCACATTTCCTACAT-3' and reverse, 5'-CCCAGTCTCAT TTAGCCACAG-3'; mouse SR-A forward, 5'-TCCTTGATTT CGTCAGTCCAG-3' and reverse, 5'-CCTCCTGTTGCTTTG CTGTAG-3'; ABCG1 forward, 5'-GCCTACTACCTGGCAA AGACC-3' and reverse, 5'-GAACAGCACAAAACGCACAG-3'; and GAPDH forward, 5'-GGTGAAGGTCGGTGTGAACG-3' and reverse, 5'-CTCGCTCCTGGAAGATGGTG-3'. The reaction of qRT-PCR was performed by the $\mathrm{iQ}^{\mathrm{TM}}$ SYBR-Green Supermix (Bio-Rad, Hercules, CA, USA) under the following conditions: $3 \mathrm{~min}$ at $95^{\circ} \mathrm{C}$ for $1 \mathrm{cycle}, 10 \mathrm{sec}$ at $95^{\circ} \mathrm{C}, 30 \mathrm{sec}$ at $60^{\circ} \mathrm{C}$ for 39 cycles, $95^{\circ} \mathrm{C}$ for $5 \mathrm{sec}$.

Western blot analysis. Nuclear extracts were prepared as described in our previous study (15). Total cell proteins were extracted as follows: cells were scraped and suspended in icecold PBS, then centrifuged at 1,000 x g for $10 \mathrm{~min}$. Cells were lysed with $180 \mu 1$ RIPA lysis buffer (Beyotime, Jiangsu, China) and vortexed every $5 \mathrm{~min}$ at $4^{\circ} \mathrm{C}$ for $30 \mathrm{~min}$. The supernatant as total cell extracts was collected after centrifuging at $12,000 \mathrm{x} \mathrm{g}$ for $15 \mathrm{~min}$ at $4^{\circ} \mathrm{C}$. All protein concentrations were determined by bicinchoninic acid protein assay kit (Biomed Biotech Co., Ltd., Beijing, China). Aliquots $(50 \mu \mathrm{g})$ of total or nuclear extracts separated on 8-12\% SDS-polyacrylamide minigels, and transferred onto nitrocellulose membranes (Amersham, Buckinghamshire, England). The membranes were incubated with $1.5 \%$ BSA and then incubated with anti-ABCA1, antiABCG1, anti-SR-BI, anti-SR-A, anti-CD36, anti-Fos, anti-c-Jun or anti- $\beta$-actin antibodies overnight at $4^{\circ} \mathrm{C}$. The protein expres-

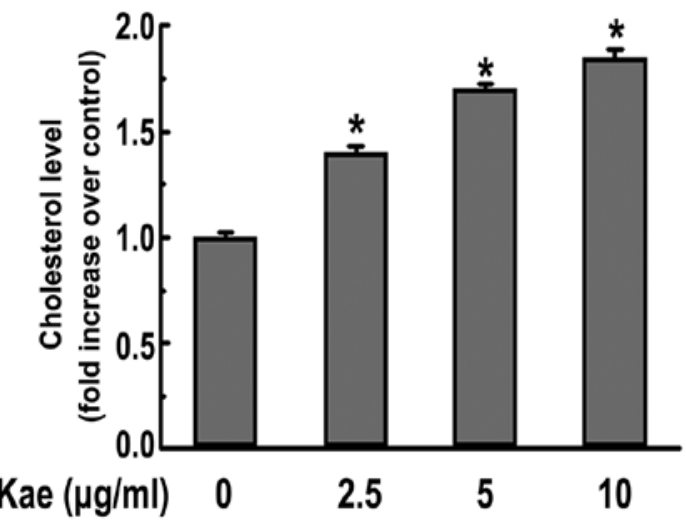

Figure 3. Kaempferol (Kae) promotes cholesterol efflux from THP-1-derived macrophages. Cells were pre-treated with various concentrations of kaempferol $(2.5,5$ and $10 \mu \mathrm{g} / \mathrm{ml})$ for $24 \mathrm{~h}$ and were then treated with NBD cholesterol $(1 \mu \mathrm{g} / \mathrm{ml})$ for an additional $6 \mathrm{~h}$ in the presence of kaempferol. Cholesterol efflux was expressed as the percentage fluorescence in the medium relative to the total fluorescence.The data are representative of 3 independent experiments (means $\pm \mathrm{SEM}$ ). ${ }^{*} \mathrm{P}<0.05$ compared with the control.

sion was detected by an enhanced chemiluminescence kit (ECL; Perkin-Elmer Life Sciences) and densitometric analysis was performed using the 720 BK/01837 System (Bio-Rad).

Statistical analysis. Data are presented as the means \pm SEM and analyzed using one-way analysis of variance (ANOVA) and the Newman-Keuls test was used to locate any significant differences identified by ANOVA. Differences were considered statistically significant when $\mathrm{P}<0.05$. All experiments were performed at least 3 times.

\section{Results}

Viability of macrophages is not affected by kaempferol at the chosen concentrations. The cytotoxicity of kaempferol at various concentrations $(2.5,5$ and $10 \mu \mathrm{g} / \mathrm{ml})$ was not obvious after $24 \mathrm{~h}$ of incubation (Fig. 2). Therefore, a concentration range of $2.5-10 \mu \mathrm{g} / \mathrm{ml}$ was chosen for subsequent experiments.

Kaempferol inhibits lipid accumulation and promotes cholesterol efflux from THP-1-derived macrophages. Lipid accumulation, a hallmark of foam cell formation, was detected in the cells treated with oxLDL in the presence or absence of kaempferol. Co-incubation with kaempferol and oxLDL significantly ameliorated intracellular lipid accumulation in the macrophages compared with the oxLDL-treated group, as revealed by Oil red $\mathrm{O}$ staining (Fig. 7). These data suggest that kaempferol suppresses oxLDL uptake and foam cell formation in macrophages. Moreover, kaempferol promoted cholesterol efflux from macrophages in a dose-dependent manner, as demonstrated by the NBD-labeled cholesterol that was used (Fig. 3). This is likely to contribute to the protective effect of kaempferol on macrophage foam cell formation.

Kaempferol increases the expression of SR-BI, ABCAI and $A B C G 1$, but decreases the expression of CD36 in macrophages. To investigate the mechanisms underlying the suppression of foam cell formation by kaempferol, ABCA1, ABCG1, SR-BI, SR-A and CD36 expression, which are reported to play critical 
A
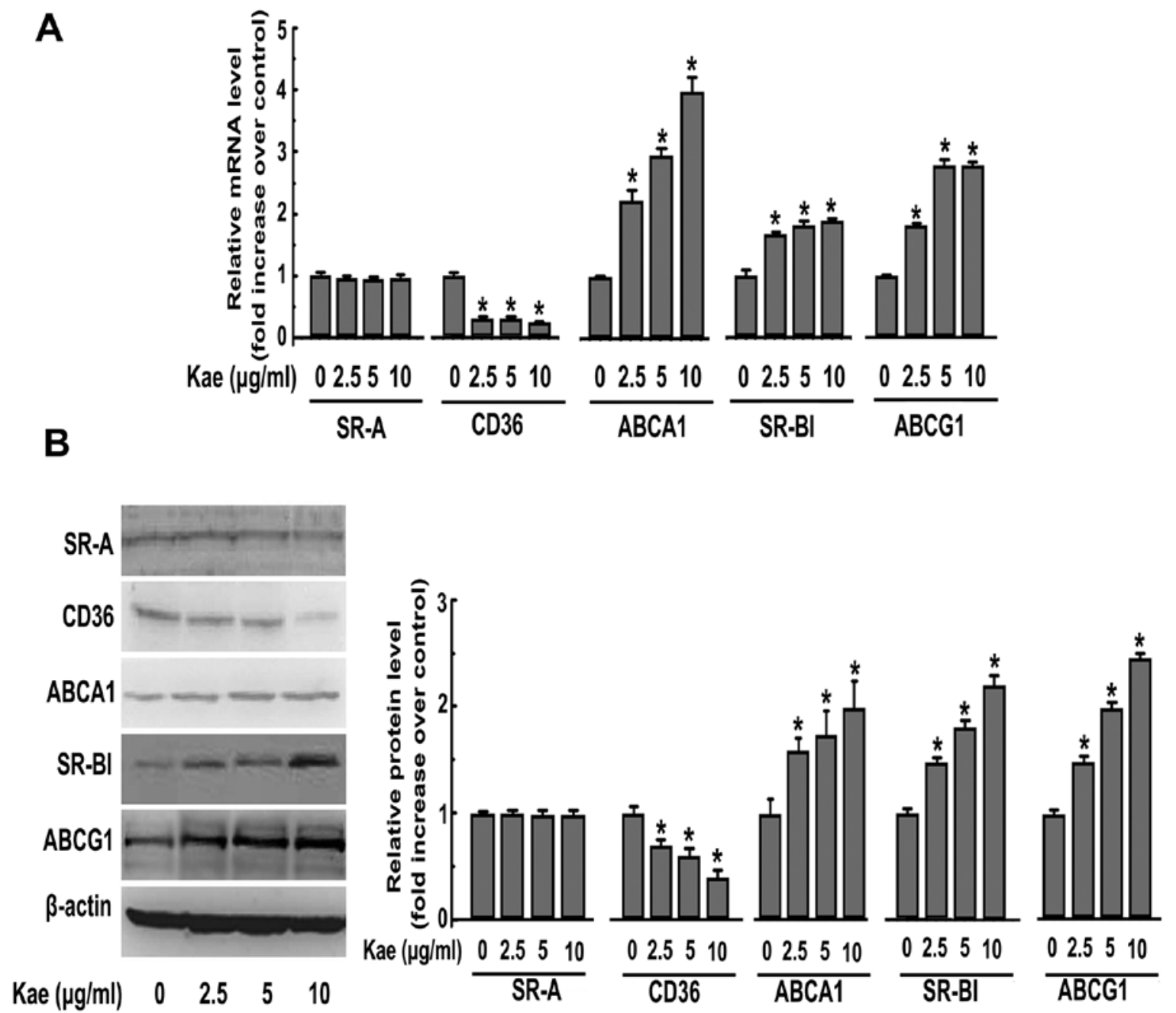

Figure 4. Kaempferol (Kae) increases the expression of SR-BI, ABCA1, ABCG1, but decreases the expression of CD36 in macrophages. (A) THP-1-derived macrophages were treated with kaempferol $(2.5,5$ and $10 \mu \mathrm{g} / \mathrm{ml})$ for $24 \mathrm{~h}$. After treatment, total RNA was extracted and then subjected to qRT-PCR to detect the mRNA expression of SR-A, CD36, ABCA1, ABCG1 and SR-BI. (B) Macrophages were incubated with various concentrations (2.5, 5 and $10 \mu \mathrm{g} / \mathrm{ml})$ of kaempferol for $24 \mathrm{~h}$. Western blot analysis was used to detect the protein expression of SR-A, CD36, ABCA1, ABCG1 and SR-BI after treatment. The data are representative of 3 independent experiments (means \pm SEM). ${ }^{*} \mathrm{P}<0.05$ compared with the control.

roles during lipid accumulation in macrophages (18), were determined following the incubation of THP-1-derived macrophages with kaempferol. Treatment with kaempferol at various concentrations $(2.5,5$ and $10 \mu \mathrm{g} / \mathrm{ml})$ for $24 \mathrm{~h}$ dose-dependently decreased the mRNA and protein expression of CD36 without affecting the expression of SR-A (Fig. 4). Additionally, the expression of ABCA1, ABCG1 and SR-BI was significantly enhanced at the protein and mRNA levels in response to kaempferol treatment (Fig. 4).

Kaempferol-induced CD36 inhibition is mediated by the $c$-Jun-AP-1 pathway. It has recently been reported that in macrophages, that c-Jun and c-Fos, 2 key subunits of AP-1, are required for the gene expression of SR-A (18). In addition, AP-1 activity contributes to the fate of the cell after kaempferol treatment (19). Therefore, in this study, we examined the possibility that the kaempferol-mediated downregulation of CD36 expression in macrophages is regulated by the AP-1 pathway. The nuclear expression of c-Fos was not significantly altered following the kaempferol treatment of THP-1-derived macrophages (Fig. 5A). However, the kaempferol treatment of macrophages was associated with dose-dependent decreases in nuclear c-Jun expression levels (Fig. 5A). Therefore, we subsequently investigated whether the inhibition of the nuclear translocation of c-Jun is critical for foam cell formation. To this end, we used the c-Jun N-terminal kinase (JNK) inhibitor, SP600125, which is a potent, cell-permeable selective and reversible inhibitor of JNK1, JNK2 and JNK3. We found that the addition of the inhibitor augmented the kaempferol-mediated effect on the downregulation of c-Jun nuclear expression (Fig. 5B), CD36 expression (Fig. 5C) and lipid accumulation in macrophages (Fig. 7). Collectively, these results suggest that the suppression of CD36 expression and subsequent alleviation of foam cell formation by kaempferol are partly c-Jun-AP-1-dependent.

Kaempferol-induced SR-BI, ABCAI and ABCG1 upregulation is mediated by $\mathrm{HO}-1$. We determined the role of $\mathrm{HO}-1$ in the kaempferol-mediated upregulation of SR-BI, ABCA1 and ABCG1. The protein expression of HO-1 in macrophages was dose-dependently elevated following kaempferol treatment as revealed by western blot analysis (Fig. 6A). Additional 
A
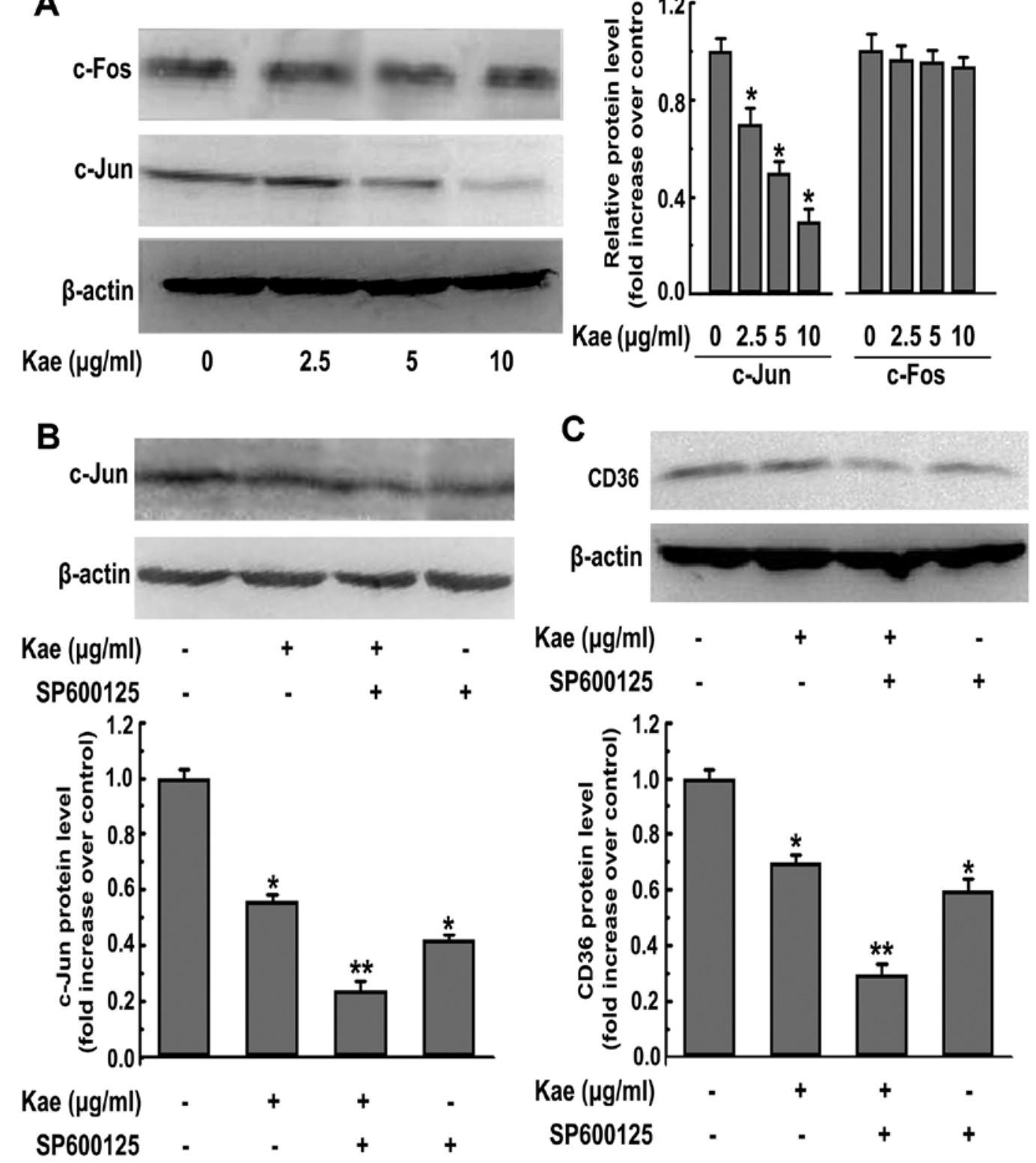

Figure 5. Nuclear translocation of c-Jun is involved in the kaempferol (Kae)-mediated suppression of CD36 expression in macrophages. (A) THP-1-derived macrophages were treated with kaempferol $(2.5,5$ and $10 \mu \mathrm{g} / \mathrm{ml})$ for $6 \mathrm{~h}$, nuclear extracts were prepared and the protein level of c-Fos, c-Jun was detected by western blot analysis. (B and C) Macrophages were pre-treated with SP600125 (10 $\mu \mathrm{M})$ for $1 \mathrm{~h}$, and were either treated or not with kaempferol (5 $\mu \mathrm{g} / \mathrm{ml})$ for (B) $6 \mathrm{~h}$ or (C) $24 \mathrm{~h}$. The nuclear expression of c-Jun and the protein expression of CD36 were determined by western blot analysis. The data are representative of 3 independent experiments (means $\pm \mathrm{SEM}$ ). $\mathrm{P}<0.05$ compared with the control; ${ }^{* * *} \mathrm{P}<0.05$ compared with the kaempferol-treated group.

experiments were then performed to determine whether HO-1 participates in the suppressive effect of kaempferol on foam cell formation. Our results showed that the transfection of HO-1 shRNA at the concentration of $400 \mathrm{ng} / \mathrm{ml}$ completely abrogated kaempferol-induced HO-1 protein expression (Fig. 6B), while the transfection of corresponding scrambled shRNA failed to do so. In addition, we found that the HO-1 shRNA transfection significantly reversed the kaempferolinduced inhibition of foam cell formation (Fig. 7). Of note, the kaempferol-mediated effects on the upregulation of SR-BI, ABCA1 and ABCG1 (Fig. 6C) and cholesterol efflux (Fig. 6D) were also reversed in the presence of HO-1 shRNA transfection. Taken together, these data indicate the involvement of HO-1 in the kaempferol-mediated protective effects on macrophage foam cells.

\section{Discussion}

A number of previous studies have demonstrated that kaempferol exerts protective effects against vascular diseases in humans as well as animals $(10,12)$. The anti-atherogenic function of kaempferol has been demonstrated. For instance, kaempferol has been shown to inhibit oxLDL-mediated apoptosis (19) and attenuate the cytokine-induced expression of vascular cell adhesion molecule-1 (VCAM-1), intercellular adhesion molecule-1 (ICAM-1) in endothelial cells (20). Kaempferol also modulates vascular dysfunction in the spontaneously hypertensive rat (21). In addition, it is well known that kaempferol alleviates the inflammatory response in macrophages (22). However, the effects of kaempferol on macrophage foam cell formation are not yet well understood. 

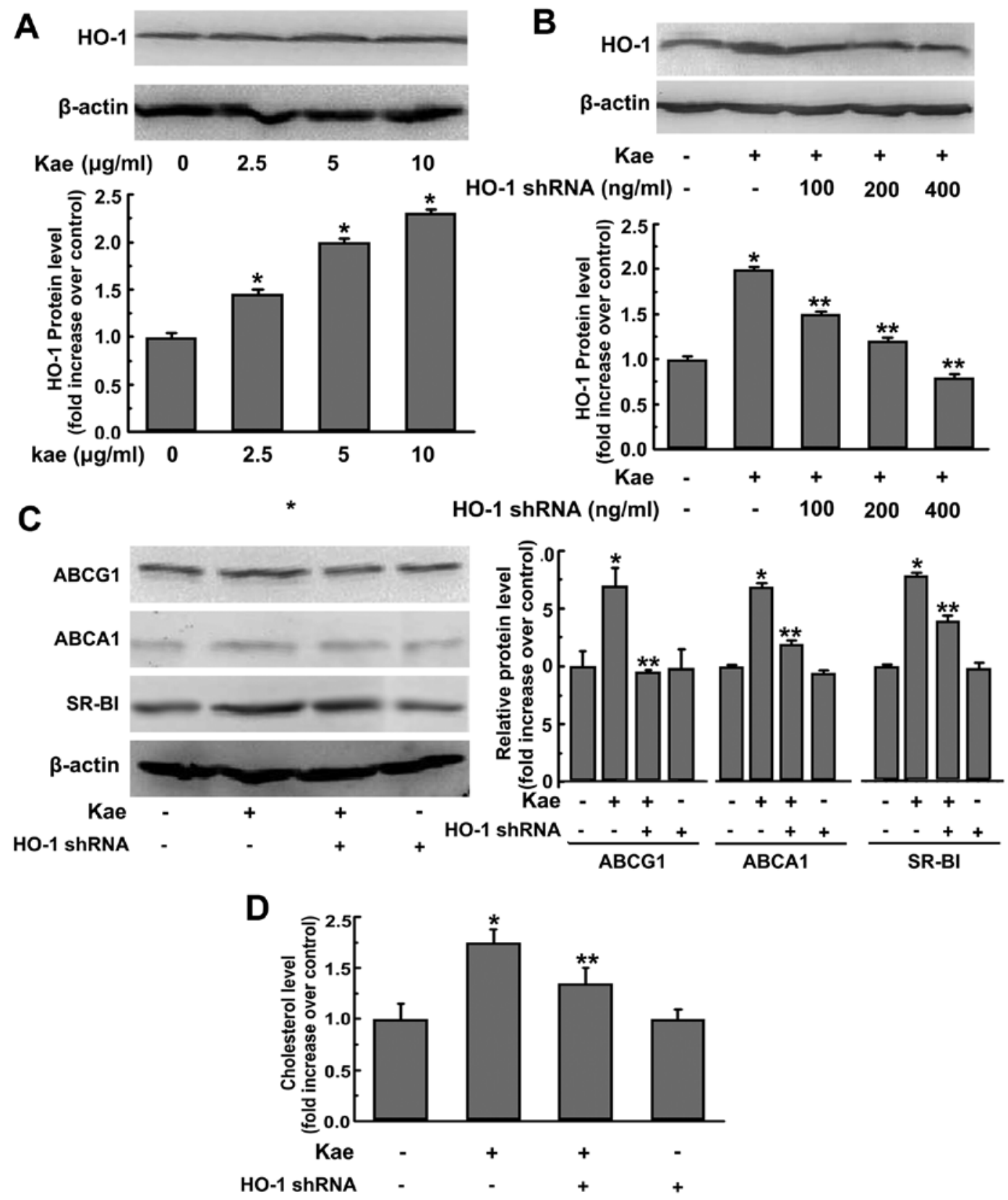

Figure 6. The kaempferol (Kae)-induced SR-BI, ABCA1 and ABCG1 upregulation is mediated by HO-1. (A) THP-1-derived macrophages were incubated with various concentrations $(2.5,5$ and $10 \mu \mathrm{g} / \mathrm{ml})$ of kaempferol for $12 \mathrm{~h}$. Western blot analysis was used to detect the protein expression of HO-1 after treatment. (B) Macrophages were transfected with HO-1 shRNA $(100,200$ and $400 \mathrm{ng} / \mathrm{ml})$ for $24 \mathrm{~h}$, and were then either treated or not with kaempferol $(5 \mu \mathrm{g} / \mathrm{ml})$ for $12 \mathrm{~h}$. The protein expression of HO-1 was determined by western blot analysis. (C and D) Macrophages were transfected with HO-1 shRNA (400 ng/ml) for $24 \mathrm{~h}$, and were then either treated or not with kaempferol $(5 \mu \mathrm{g} / \mathrm{ml})$ for an additional $24 \mathrm{~h}$. The protein expression of SR-BI, ABCA1, ABCG1 was determined by western blot analysis. Cholesterol efflux was analyzed as described in Materials and methods. The data are representative of 3 independent experiments (means $\pm \mathrm{SEM}$ ). ${ }^{*} \mathrm{P}<0.05$ compared with the control; ${ }^{* *} \mathrm{P}<0.05$ compared with the kaempferol-treated group.

Uncontrolled oxLDL uptake and impaired cholesterol efflux are reported to be the major contributors of foam cell formation (17). Of note, our data suggest that kaempferol ameliorates oxLDL-induced foam cell formation by reducing lipid accumulation and promoting cholesterol efflux from macrophage, confirming the findings reported in the study by Tu et al (23). Kaempferol suppressed oxLDL uptake by THP-1-derived macrophages, as revealed by flow cytometry analysis. We thus performed experiments to further examine the molecular mechanisms underlying the beneficial function of kaempferol during foam cell formation.
Macrophage SRs, such as CD36 and SR-A, have previously been implicated in foam cell formation and atherogenesis, during which these receptors participate in the uptake of oxLDL (24). However, it is not yet well known whether similar mechanisms are involved in the kaempferol-mediated protection in macrophage foam cell formation. In the present study, we showed that kaempferol significantly suppressed the mRNA and protein expression of CD36 without affecting the expression of SR-A. The genetic inactivation of $\mathrm{CD} 36$ has previously been shown to reduce oxLDL uptake in vitro and in atherosclerotic lesions in mice (25), strongly supporting a pro-atherogenic 
A

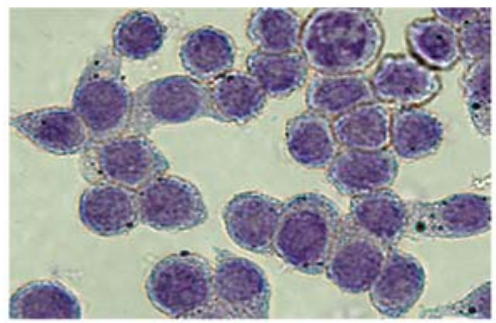

C

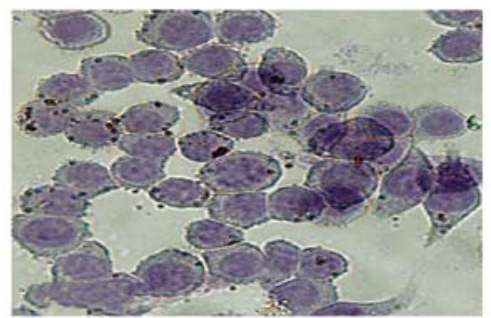

D

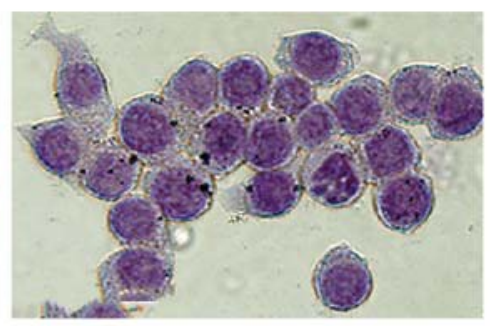

B

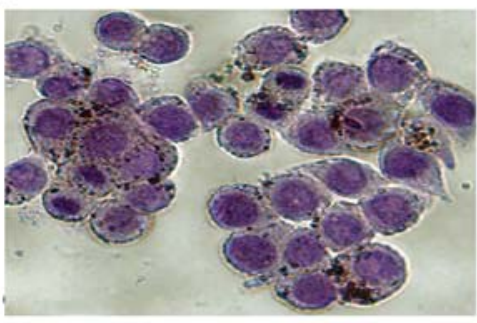

E

Figure 7. The AP-1 and HO-1 pathway functionally participate in the kaempferol (Kae)-mediated protective effects on macrophage foam cells. THP-1-derived macrophages were incubated for $1 \mathrm{~h}$ with SP600125 $(10 \mu \mathrm{M})$ or transfected with HO-1 shRNA (400 ng/ml) for $24 \mathrm{~h}$ respectively, and were then treated with oxLDL in the presence or absence of kaempferol $(5 \mu \mathrm{g} / \mathrm{ml})$ for an additinoal $24 \mathrm{~h}$. (A) Control group; (B) the oxLDL-treated group; (C) the oxLDL plus kaempferol group; (D) the oxLDL plus SP600125 group; and (E) the oxLDL plus HO-1 shRNA group. The cells with various treatments were fixed and then stained with Oil red O. The magnification was $\mathrm{x} 400$.

role for CD36. In view of CD36 function, it may be concluded that the decrease in CD36 expression followed by kaempferol treatment may contribute to the reduced lipid accumulation and subsequent inhibition of foam cell formation.

To our knowledge, our study, for the first time identifies c-Jun-AP-1 as the key transcriptional factor for the kaempferolinduced downregulation of CD36 expression. c-Fos and c-Jun are 2 dimers of AP-1. We demonstrated that the nuclear translocation of c-Fos was not affected by kaempferol. However, kaempferol significantly attenuated the nuclear translocation of c-Jun, which is involved in the induction of CD36 (26). This notion is also supported by the results that the inhibition of AP-1 increases the effects of kaempferol on the downregulation of CD36 expression and the inhibition of foam cell formation. This finding is consistent with previous observations in a study of the downregulation of CD36 by $1,25(\mathrm{OH}) 2$ vitamin $\mathrm{D}(27)$.

In addition to its effect on CD36 expression, we further investigated the effect of kaempferol on the expression of ABCA1, ABCG1 and SR-BI, 3 major transporters for cholesterol efflux from macrophage foam cells. Duh et al reported that kaempferol increases ABCA1 and SR-BI expression in RAW264.7 macrophages (28), and similar results were obtained in THP-1-derived macrophages in this study. Moreover, we found that ABCG1 expression was also increased in response to kaempferol treatment. It is well established that ABCA1, ABCG1 and SR-BI play a critical role in the cholesterol homeostasis in macrophages (29). Studies using individual transporter-deficient animals showed that atherosclerotic lesions and foam cell accumulation were markedly promoted (17). Recent studies have indicated that the elevated function of RCTs in macrophages, leading to reduced deposition of cholesterol in macrophages, by several dietary flavonoids with anti-atherogenic properties, such as quercetin, EGb 761 and daidzein and resveratrol $(18,30,31)$. In view of their function, the upregulation of ABCA1, ABCG1 and SR-BI expression following kaempferol treatment observed in the current study is likely to promote the cholesterol efflux and subsequent inhibits foam cell formation. More importantly, our results suggest that the induced expression of ABCA1, SR-BI and ABCG1 by kaempferol is accompanied by increased HO-1 expression. This finding was further supported by the results from western blot analysis, in which the kaempferol-mediated upregulation of ABCA1, SR-BI and ABCG1 was reversed by the knockdown of the HO-1 gene using shRNA. In addition, the inhibition of HO-1 attenuated the kaempferol effects on the upregulation of cholesterol efflux and the downregulation of lipid accumulation in macrophages. These results suggest that the expression of HO-1 is required for the induction of ABCA1, SR-BI and ABCG1 expression by kaempferol. Our findings are in agreement with reports that the exogenous overexpression of HO-1 using agonist or adenovirus retards the progression of atherosclerosis in hyperlipidaemic mice $(32,33)$. Although the detailed mechanisms of how kaempferol affects the functions of HO-1 remain to be further investigated, in this study, we discovered a unique pathway for the kaempferol-mediated upregulation of ABCA1, SR-BI and ABCG1. A number of studies have reported that bilirubin or carbon monoxide regulate the antioxidative or anti-inflammatory action of HO-1 $(32,34)$. The involvement of this pathway in the kaempferol-elicited induction of ABCA1, SR-BI and ABCG1 expression remains to be investigated in the future.

In conclusion, our study provides new insights into the atheroprotective nature of kaempferol in THP-1-derived macrophages, which promotes cholesterol efflux and reduces lipid accumulation in foam cells via the regulation of ABCA1, SR-BI, ABCG1 and CD36 expression. CD36 is downregulated by kaempferol by inhibiting c-Jun nuclear translocation, whereas ABCA1, SR-BI, ABCG1 expression is upregulated following kaempferol treatment through the enhanced protein expression of HO-1. The findings of this study provide a novel 
explanation for the anti-atherogenic properties of kaempferol, as well as possible molecular targets which may be used for the development of therapeutic strategies for the treatment of atherosclerosis.

\section{Acknowledgements}

This study was supported by a grant from the Public Health Bureau of Chongqing, China (no. 2009-1-6).

\section{References}

1. Tsimikas S, Miyanohara A, Hartvigsen K, et al: Human oxidationspecific antibodies reduce foam cell formation and atherosclerosis progression. J Am Coll Cardiol 58: 1715-1727, 2011.

2. Sung HJ, Kim J, Kim Y, Jang SW and Ko J: N-acetyl cysteine suppresses the foam cell formation that is induced by oxidized low density lipoprotein via regulation of gene expression. Mol Biol Rep 39: 3001-3007, 2012.

3. Li AC and Glass CK: The macrophage foam cell as a target for therapeutic intervention. Nat Med 8: 1235-1242, 2002.

4. Kleemann R, Zadelaar S and Kooistra T: Cytokines and atherosclerosis: a comprehensive review of studies in mice. Cardiovasc Res 79: 360-376, 2008

5. Hrboticky N, Draude G, Hapfelmeier G, Lorenz R and Weber PC: Lovastatin decreases the receptor-mediated degradation of acetylated and oxidized LDLs in human blood monocytes during the early stage of differentiation into macrophages. Arterioscler Thromb Vasc Biol 19: 1267-1275, 1999.

6. Wang X and Rader DJ: Molecular regulation of macrophage reverse cholesterol transport. Curr Opin Cardiol 22: 368-372, 2007.

7. Cuchel $\mathrm{M}$ and Rader DJ: Macrophage reverse cholesterol transport: key to the regression of atherosclerosis? Circulation 113: 2548-2555, 2006

8. Chirumbolo S: The role of quercetin, flavonols and flavones in modulating inflammatory cell function. Inflamm Allergy Drug Targets 9: 263-285, 2010.

9. González-Gallego J, Sánchez-Campos S and Tuñón MJ: Antiinflammatory properties of dietary flavonoids. Nutr Hosp 22: 287-293, 2007.

10. Calderón-Montaño JM, Burgos-Morón E, Pérez-Guerrero C and López-Lázaro M: A review on the dietary flavonoid kaempferol. Mini Rev Med Chem 11: 298-344, 2011.

11. Park MY, Ji GE and Sung MK: Dietary kaempferol suppresses inflammation of dextran sulfate sodium-induced colitis in mice. Dig Dis Sci 57: 355-363, 2012.

12. Xiao HB, Lu XY, Sun ZL and Zhang HB: Kaempferol regulates OPN-CD44 pathway to inhibit the atherogenesis of apolipoprotein E deficient mice. Toxicol Appl Pharmacol 257: 405-411, 2011.

13. Hong JT, Yen JH, Wang L, Lo YH, Chen ZT and Wu MJ: Regulation of heme oxygenase-1 expression and MAPK pathways in response to kaempferol and rhamnocitrin in PC12 cells. Toxicol Appl Pharmacol 237: 59-68, 2009.

14. Chen CC, Chow MP, Huang WC, Lin YC and Chang YJ: Flavonoids inhibit tumor necrosis factor-alpha-induced upregulation of intercellular adhesion molecule-1 (ICAM-1) in respiratory epithelial cells through activator protein-1 and nuclear factorkappaB: structure-activity relationships. Mol Pharmacol 66: 683-693, 2004

15. Li XY, He JL, Liu HT, Li WM and Yu C: Tetramethylpyrazine suppresses interleukin-8 expression in LPS-stimulated human umbilical vein endothelial cell by blocking ERK, p38 and nuclear factor-kappaB signaling pathways. J Ethnopharmacol 125: 83-89, 2009.

16. Kalayoglu MV, Hoerneman B, LaVerda D, Morrison SG, Morrison RP and Byrne GI: Cellular oxidation of low-density lipoprotein by Chlamydia pneumoniae. J Infect Dis 180: 780-790, 1999.
17. Cheng LC, Su KH, Kou YR, et al: $\alpha$-Lipoic acid ameliorates foam cell formation via liver $\mathrm{X}$ receptor $\alpha$-dependent upregulation of ATP-binding cassette transporters A1 and G1. Free Radic Biol Med 50: 47-54, 2011.

18. Tsai JY, Su KH, Shyue SK, et al: EGb761 ameliorates the formation of foam cells by regulating the expression of SR-A and ABCA1: role of haem oxygenase-1. Cardiovasc Res 88: 415-423, 2010.

19. Lim $\mathrm{H}$ and Kim HP: Inhibition of mammalian collagenase, matrix metalloproteinase-1, by naturally-occurring flavonoids. Planta Med 73: 1267-1274, 2007.

20. Crespo I, García-Mediavilla MV, Gutiérrez B, SánchezCampos S, Tuñón MJ and González-Gallego J: A comparison of the effects of kaempferol and quercetin on cytokine-induced pro-inflammatory status of cultured human endothelial cells. $\mathrm{Br}$ J Nutr 100: 968-976, 2008.

21. Abeywardena MY and Head RJ: Dietary polyunsaturated fatty acid and antioxidant modulation of vascular dysfunction in the spontaneously hypertensive rat. Prostaglandins Leukot Essent Fatty Acids 65: 91-97, 2001.

22. Mekhora C, Muangnoi C, Chingsuwanrote P, Dawilai S, Svasti S, Chasri K and Tuntipopipat S: Eryngium foetidum suppresses inflammatory mediators produced by macrophages. Asian Pac J Cancer Prev 13: 653-664, 2012.

23. Tu YC, Lian TW, Yen JH, Chen ZT and Wu MJ: Antiatherogenic effects of kaempferol and rhamnocitrin. J Agric Food Chem 28: 9969-9976, 2007.

24. Witztum JL: You are right too! J Clin Invest 115: 2072-2075, 2005.

25. Kunjathoor VV, Febbraio M, Podrez EA, et al: Scavenger receptors class A-I/II and CD36 are the principal receptors responsible for the uptake of modified low density lipoprotein leading to lipid loading in macrophages. J Biol Chem 277: 49982-49988, 2002.

26. Katayama I, Hotokezaka Y, Matsuyama T, Sumi T and Nakamura T: Ionizing radiation induces macrophage foam cell formation and aggregation through JNK-dependent activation of CD36 scavenger receptors. Int J Radiat Oncol Biol Phys 70: 835-846, 2008

27. Oh J, Weng S, Felton SK, et al: $1,25(\mathrm{OH}) 2$ vitamin d inhibits foam cell formation and suppresses macrophage cholesterol uptake in patients with type 2 diabetes mellitus. Circulation 120: 687-698, 2009.

28. Duh PD, Hsiao WC and Wang BS: An aqueous extract of Welsh onion green leaves increase ABCA1 and SR-BI expression in macrophage RAW 264.7 cells. Food Chemistry 107: 1029-1038, 2008.

29. Lu KY, Ching LC, Su KH, et al: Erythropoietin suppresses the formation of macrophage foam cells: role of liver $\mathrm{X}$ receptor alpha. Circulation 121: 1828-1837, 2010

30. Chang YC, Lee TS and Chiang AN: Quercetin enhances ABCA1 expression and cholesterol efflux through a p38-dependent pathway in macrophages. J Lipid Res 53: 1840-1850, 2012.

31. Gao J, Xu Y, Yang Y, et al: Identification of upregulators of human ATP-binding cassette transporter A1 via high-throughput screening of a synthetic and natural compound library. J Biomol Screen 13: 648-656, 2008.

32. Ishikawa K, Sugawara D, Wang Xp, Suzuki K, Itabe H, Maruyama Y and Lusis AJ: Heme oxygenase-1 inhibits atherosclerotic lesion formation in ldl-receptor knockout mice. Circ Res 88: 506-512, 2001.

33. Juan SH, Lee TS, Tseng KW, Liou JY, Shyue SK, Wu KK and Chau LY: J Adenovirus-mediated heme oxygenase-1 gene transfer inhibits the development of atherosclerosis in apolipoprotein E-deficient mice. Circulation 104: 1519-1525, 2001.

34. Idriss NK, Blann AD and Lip GY: Hemoxygenase-1 in cardiovascular disease. J Am Coll Cardiol 52: 971-978, 2008. 